\title{
ORIGINAL RESEARCH \\ Stenting from the Vertebral Artery to the Posterior Inferior Cerebellar Artery
}

\author{
M.J. Kim \\ J. Chung \\ BACKGROUND AND PURPOSE: There are only a few reports on the feasibility and safety of stents used \\ in the PICA, and clinical and angiographic follow-up results have not been fully addressed. We report \\ our experiences of treating PICA origin or vertebral artery-PICA lesions by using self-expanding stents \\ as adjuvant or rescue therapy with angiographic and clinical follow-up results. \\ H.G. Roh
}

MATERIALS AND METHODS: Six patients were treated with self-expanding stent placements from the

B.J. Kwon

B.-s. Kim vertebral artery to the PICA. Two patients had a vertebral artery dissecting aneurysm involving the PICA origin, 3 had vertebral artery-PICA aneurysms, and 1 had segmental stenosis of the vertebral artery harboring the origin of the PICA. The safety, feasibility, and follow-up angiographic results were

T.H. Kim retrospectively evaluated.

B.M. Kim

Y.S. Shin

RESULTS: All procedures were successfully performed without any procedure-related complications. None of the patients showed PICA territorial infarction on DWI posttreatment. All patients were neurologically intact during the clinical follow-up of 3-24 months following the procedure. Follow-up angiography was performed at between 6 and 12 months in 5 of the 6 patients and was scheduled for the sixth patient but was not performed. The PICA showed good patency without in-stent stenosis in all 5 patients.

CONCLUSIONS: In patients with lesions of the PICA origin or vertebral artery-PICA lesions, vertebral artery-to-PICA stent placement may be an option for preserving PICA patency in selected cases.

ABBREVIATIONS EVT = endovascular treatment; PCA $=$ posterior cerebral artery

V ascular lesions of the vertebral artery and/or PICA, such as vertebral artery dissections, are not amenable to a surgical or endovascular approach. Especially in the treatment of lesions involving the PICA origin, precautions should be taken against the possible risk of PICA occlusion because of the risk of ischemic complications in the eloquent brain stem territory. ${ }^{1}$ If PICA flow compromise was expected or inevitable during or after treatment, revascularization of the PICA should be considered. Nevertheless, bypass surgery of the posterior circulation carries a considerable operative risk due to anastomosis in the deep and narrow operative corridor near the brain stem and cranial nerves. ${ }^{2}$ Therefore, endovascular therapies by using only placement or coiling are often considered the first-line of treatment.

Recently, user-friendly, self-expanding intracranial stents have become available for the treatment of cerebral aneurysms and intracranial atherosclerotic disease. ${ }^{3,4}$ In some circumstances, operators use those devices beyond the indications for use in the device manual. ${ }^{5}$ In particular, the use of stent devices

Received May 2, 2011; accepted after revision May 15

From the Departments of Neurosurgery (M.J.K., Y.S.S.) and Radiology (B.-S.K., T.H.K.), Seoul St. Mary's Hospital, Catholic University of Korea, Seoul, Republic of Korea; Department of Neurosurgery (J.C.), Inha University School of Medicine, Incheon, Republic of Korea; Department of Neurosurgery (S.L.K.), Bucheon St. Mary's Hospital, Catholic University of Korea, Bucheon, Republic of Korea; Department of Radiology (H.G.R.), Konkuk University Medical Center of Korea, Seoul, Republic of Korea; Department of Radiology (B.J.K.), Myongji Hospital of Korea, Goyang, Republic of Korea; and Department of Radiology (B.M.K,), Severance Hospital, Yonsei University College of Medicine, Seoul, Republic of Korea

Please address correspondence to Yong-Sam Shin, MD, Ph.D, Department of Neurosurgery, Seoul St. Mary's Hospital, Catholic University of Korea, 505 Banpo-Dong, Seocho-Gu, Seoul, Korea, 137-701; e-mail: nsshin@catholic.ac.kr

Indicates open access to non-subscribers at www.ajnr.org

http://dx.doi.org/10.3174/ajnr.A2741 in small intracranial vessels of $\leq 2-\mathrm{mm}$ diameter is still controversial. ${ }^{4,6}$ We reported the first case of vertebral artery-PICA stent placement for the preservation of patency of the PICA. ${ }^{7}$ Thereafter, we experienced 5 more cases in which vertebral artery-PICA stent placement was necessary. The purpose of this study was to report the feasibility, safety, and angiographic and clinical follow-up results of vertebral artery-PICA stent placement for various lesions in the 6 selected patients.

\section{Materials and Methods}

Between 2009 and 2010 in 3 referring hospitals in Korea, we performed stent placement in the vertebral artery-PICA in 6 cases. The 6 patients, 4 men and 2 women, underwent placement of the selfexpanding intracranial stent from the vertebral artery to the PICA. The ages of the patients ranged from 49 to 68 years with a mean of 54.8 years. Two of the 6 patients had a ruptured vertebral artery dissecting aneurysm involving the PICA origin. Three patients had vertebral artery-PICA aneurysms, and the last patient had segmental stenosis of the vertebral artery harboring the origin of the PICA. All patients underwent DSA and rotational angiography with $3 \mathrm{D}$ reconstructions to characterize the lesion and parent artery anatomy. All procedures were performed with the patient under general anesthesia. For the ruptured dissection and vertebral artery-PICA aneurysm, dual antiplatelets (aspirin, $300 \mathrm{mg}$, and clopidogrel, $300 \mathrm{mg}$ ) were administered just after the procedure and maintained for 1 year. In 5 patients, we used the Enterprise stent (Cordis, Miami Lakes, Florida) from the proximal vertebral artery to the PICA, and the Solitaire AB (ev3, Irvine, California) was used in 1 patient. Follow-up angiography was performed in 5 of the 6 patients between 6 and 12 months after the procedure, and clinical follow-up was performed from 3 to 24 months after the procedure in all patients. Clinical outcomes were evaluated by using the Glasgow Outcome Score. 


\begin{tabular}{|c|c|c|c|c|c|c|c|}
\hline \multicolumn{8}{|c|}{ Summary of characteristics of 6 patients who underwent stenting of vertebral artery-PICA } \\
\hline $\begin{array}{l}\text { Patient } \\
\text { No. }\end{array}$ & $\begin{array}{l}\text { Age }(y r) / \\
\text { Sex }\end{array}$ & Presentation & Diagnosis & Treatment & $\begin{array}{l}\text { Stent } \\
(\mathrm{mm})\end{array}$ & $\begin{array}{l}\text { Imaging/Clinical } \\
\text { F/U (mo) }\end{array}$ & GOS \\
\hline $1^{\mathrm{a}}$ & $58 / \mathrm{F}$ & Coexisting & PICA aneurysm & Stent coil & $\begin{array}{l}\text { Enterprise } \\
4.5 \times 22\end{array}$ & $11 / 24$ & 5 \\
\hline 2 & 49/M & SAH & Vertebral artery dissection & $\begin{array}{l}\text { Vertebral artery occlusion } \\
\text { with stenting }\end{array}$ & $\begin{array}{l}\text { Enterprise } \\
4.5 \times 37\end{array}$ & $12 / 23$ & 5 \\
\hline 3 & $50 / \mathrm{M}$ & Headache & Vertebral artery dissection & $\begin{array}{l}\text { Vertebral artery occlusion } \\
\text { with stenting }\end{array}$ & $\begin{array}{l}\text { Enterprise } \\
4.5 \times 22\end{array}$ & $6 / 6$ & 5 \\
\hline 4 & $55 / \mathrm{M}$ & Dizziness & Vertebral artery-PICA stenosis & Stent & $\begin{array}{l}\text { Enterprise } \\
4.5 \times 28\end{array}$ & $6 / 6$ & 5 \\
\hline 5 & 49/M & SAH & PICA aneurysm & Stent coil & $\begin{array}{l}\text { Solitaire } \\
4 \times 15\end{array}$ & $6 / 6$ & 5 \\
\hline $6^{a}$ & $68 / F$ & Coexisting & PICA aneurysm & Stent coil & $\begin{array}{l}\text { Enterprise } \\
4.5 \times 22\end{array}$ & $1 / 3$ & 5 \\
\hline
\end{tabular}

Note:-F/U indicates Follow-up; GOS, Glasgow Outcome Score.

an patient 2, "coexisting" means that there is another asymptomatic lesion.
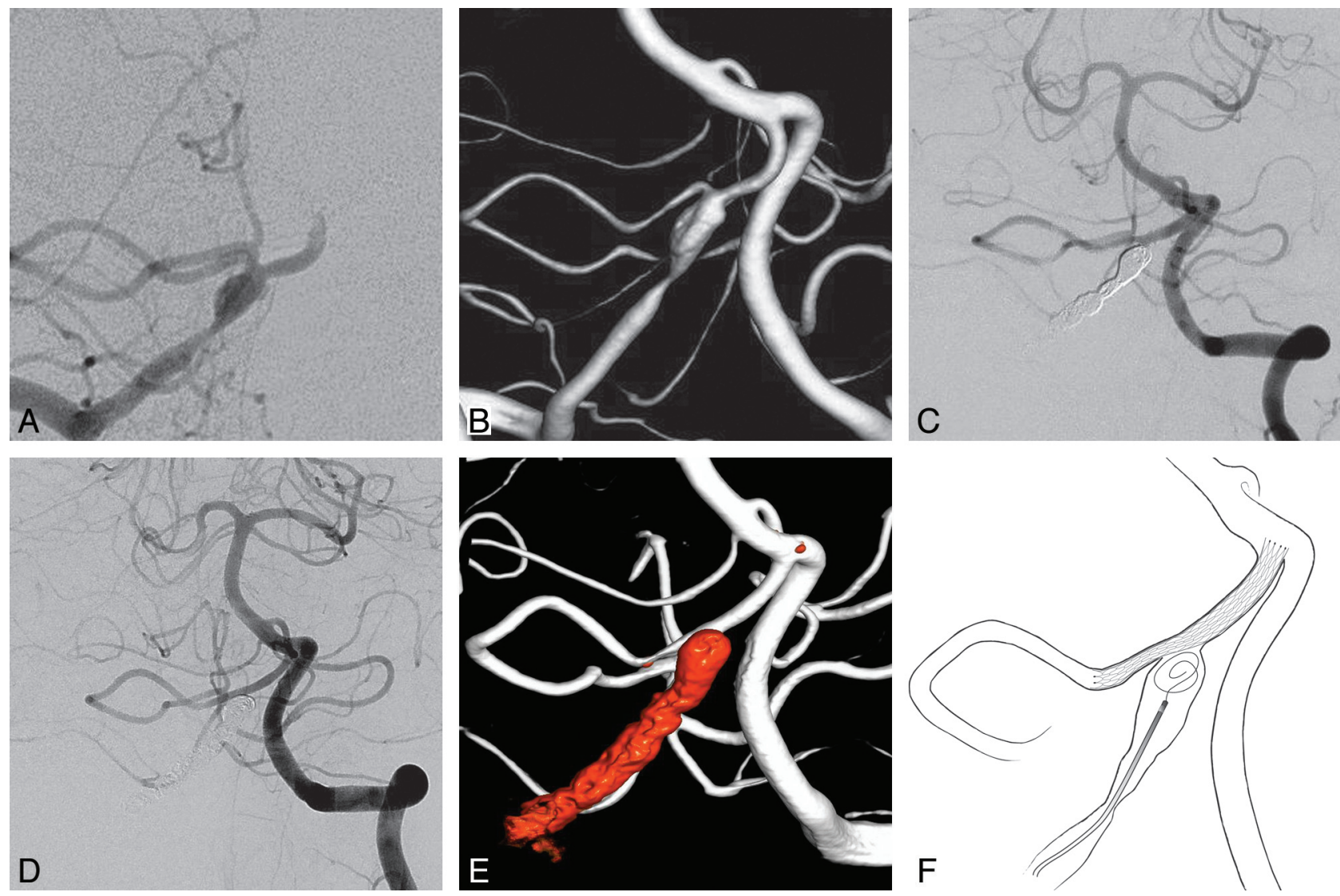

Fig 1. $A$ and $B$, The anteroposterior view of the right vertebral artery angiography $(A)$ and the $3 D$ reconstruction image $(B)$. $C$, The Enterprise stent is placed through the PICA to the distal portion of right vertebral artery via left the vertebral artery approach. $D$, The 6-month follow-up angiography reveals good PICA patency. E, The 3D reconstruction image also shows good PICA patency. F, Illustration of the vertebral artery-to-PICA stent placement with coil embolization.

\section{Results}

The characteristics of the 6 vertebral artery-PICA stent placements are summarized in the Table. All procedures were successfully performed without any complications. No patient had PICA territorial infarction on DWI posttreatment. All 6 patients were neurologically intact during the clinical follow-up from 3 to 24 months. Follow-up angiography was performed in 5 of 6 patients at $6-12$ months after the procedure. Follow-up angiography was also scheduled for the last patient but was not completed. Follow-up angiography revealed good PICA patency without in-stent stenosis in all 5 patients.

\section{Representative Cases}

Case 1. A 50-year-old man (patient 3 in the Table) was admitted with severe headache. Brain CTA revealed suspicious subarachnoid hemorrhage on the cerebellomedullary cistern and fusiform dilation of the right vertebral artery. DSA revealed a right vertebral artery dissection involving the PICA origin (Fig $1 A,-B$ ). The diameter of the right vertebral artery was $2.35 \mathrm{~mm}$, and the diameter of the PICA was $1.54 \mathrm{~mm}$. We strongly suspected the rupture of the vertebral artery dissection from the clinical symptoms of the patient. Because the patient had good flow from the contralateral vertebral artery, 
we decided to occlude the dissecting segment by endovascular treatment. However, the origin of the PICA involved the distal segment of dissection. Therefore, we placed the self-expanding stent through the vertebral artery to the PICA, approached from the contralateral vertebral artery. A 5F Envoy guiding catheter (Cordis) was introduced into both vertebral arteries (V2 segment), and then a microcatheter (Prowler Select Plus, Cordis) was placed in the distal portion of the right PICA through the left vertebral artery. A $4.5 \times 22 \mathrm{~mm}$ Enterprise stent was then placed through the PICA to the right distal vertebral artery. Another microcatheter (Excelsior 1018; Boston Scientific, Natick, Massachusetts) was placed at the dissecting segment from a right vertebral artery approach.

The dissected segment of the vertebral artery was completely occluded by coil embolization (Fig 1C). During the coiling, 2000 IU of intravenous heparin was administered. The right vertebral artery was completely occluded, and the flow from the vertebral artery to the PICA was preserved by flow from the left vertebral artery. Before the procedure, dual antiplatelet loading (aspirin, $200 \mathrm{mg}$, and clopidogrel, $300 \mathrm{mg}$ ) was performed, and just after the procedure, dual antiplatelets (aspirin, $100 \mathrm{mg}$, and clopidogrel, $75 \mathrm{mg}$ per day) were maintained. The diffusion MR imaging showed a few small embolic infarctions around the PICA, but the patient showed no neurologic symptoms. The 1-week follow-up angiography showed good patency of the PICA, and the patient was discharged without neurologic deficits. The 6-month follow-up angiography showed that the dissected segment was completely occluded (Fig 1D). The caliber of the PICA increased slightly $(1.66 \mathrm{~mm})$ with good patency as the PICA was straightened and the vertebral artery-PICA angle became larger. In addition, the patient had no neurologic deficit at the 6-month clinical follow-up.

Case 2. A 55-year-old man (patient 4 in the Table) presenting with repetitive dizziness was referred from the department of neurology. The patient had been taking dual antiplatelets (aspirin, $100 \mathrm{mg}$, and clopidogrel, $75 \mathrm{mg}$ ) for stenosis of both vertebral arteries. DSA revealed a left vertebral artery stenosis involving the origin of the PICA (Fig $2 A,-B$ ). DSA showed slight interval progression of focal segmental stenosis involving the left vertebral artery, just proximal to the origin of the PICA compared with the previous study. The diameter of the most stenotic vertebral artery was $1.11 \mathrm{~mm}$, and the diameter of the proximal PICA was $1.27 \mathrm{~mm}$.

The patient had several recurrent ischemic attacks despite proper antiplatelet medications, and the follow-up angiography showed aggravation of vertebral artery stenosis. We decided to perform vertebral artery stent placement for treatment of the vertebral artery stenosis. After giving intravenous heparin (3000 IU), a preshaped 45-degree microcatheter (Excelsior SL-10, Boston Scientific) was place in the basilar artery. After that, the microcatheter was removed by using an exchange microwire, and a $3.0 \times 15 \mathrm{~mm}$ intracranial balloon (Gateway, Boston Scientific) was placed at the stenotic lesion of the vertebral artery. Then, angioplasty (6 atm) was performed. Post-balloon angiography showed dilation of stenosis involving the vertebral artery, with the remaining stenosis involving the PICA origin. A $4.5 \times 20 \mathrm{~mm}$ Wingspan stent (Boston Scientific) was then placed at the vertebral artery across the origin of the PICA.
Poststent angiography showed favorable anterograde flow to the vertebral artery, but without contrast filling in the PICA. We gave intravenous heparin (1000 IU) and intra-arterial tirofiban (1 $\mathrm{mg}$ ) immediately, but repetitive angiography showed no more contrast filling of the PICA (Fig 2C). We suspected "snowplowing" of the PICA by placement of the stent in the vertebral artery. A preshaped $90^{\circ}$ microcatheter (Excelsior SL-10) was placed in the PICA; then, it was exchanged with the microcatheter (Prowler Select Plus). A $4.5 \times$ $28 \mathrm{~mm}$ Enterprise stent was placed from the proximal PICA to the vertebral artery, and the flow to the PICA was completely recovered.

We confirmed good patency of the vertebral artery and the PICA flow with follow-up angiography after 30 minutes. Just after the procedure, dual antiplatelets (aspirin, $100 \mathrm{mg}$, and clopidogrel, $75 \mathrm{mg}$ per day) were maintained. Diffusion MR imaging showed no infarction at the territory of the PICA, and the patient was discharged without neurologic deficits. The 6-month follow-up angiography showed that the vertebral artery and PICA had good patency without restenosis (Fig 2D). The diameter of the poststenting PICA increased slightly to $1.39 \mathrm{~mm}$. The patient displayed no neurologic deficit on the 6-month clinical follow-up.

\section{Discussion}

Treatment of vertebral artery lesions involving the PICA origin requires complete resolution of the vertebral artery lesion, maintaining PICA patency or revascularization of the PICA by surgical procedures such as an occipital artery-to-PICA bypass, ${ }^{8}$ PICA side-to-side anastomosis ${ }^{9}$ or PICA transposition. ${ }^{10}$ However, these surgical strategies may be inconvenient because they may take a relatively longer time and result in some postoperative complications, such as lower cranial nerve palsy. ${ }^{11}$ With EVT, the choice of treatment for patients with vertebral artery-PICA lesions depends on the patency of the contralateral vertebral artery as well as the relationship between the vertebral artery lesion and the location of the PICA origin. $^{7}$ If the vertebral artery dissection/aneurysm is located proximal or distal to the PICA without hypoplasia of the opposite vertebral artery, the lesion can be treated easily with total occlusion of the lesion segment so that the PICA will be filled from the ipsilateral or contralateral vertebral artery. ${ }^{8}$

In case 1 (patient 3 in the Table), the rupture point of the vertebral artery dissection was adjacent to the origin of the PICA. There was considerable risk of PICA occlusion if we performed complete occlusion of the dissecting segment. Considering the surgical treatment, we would plan to trap the dissected segment with an occipital artery-PICA bypass. In place of this surgical strategy, we considered EVT by using any stent based on previous experiences of vertebral artery-PICA stent placement. ${ }^{7}$ The PICA could be occluded by coil embolization in the proximal segment of dissection, so we concluded that the proximal vertebral artery-to-PICA stent placement might prevent an overcoiling or snowplowing with preservation of the PICA patency. Then we performed coil embolization of the vertebral artery dissection after stent placement of the vertebral artery-to-PICA (Fig 1).

In case 2 (patient 4 in table), undesired obliteration of the PICA flow occurred after vertebral artery stent placement. We thought that this event also occurred because of snowplowing 

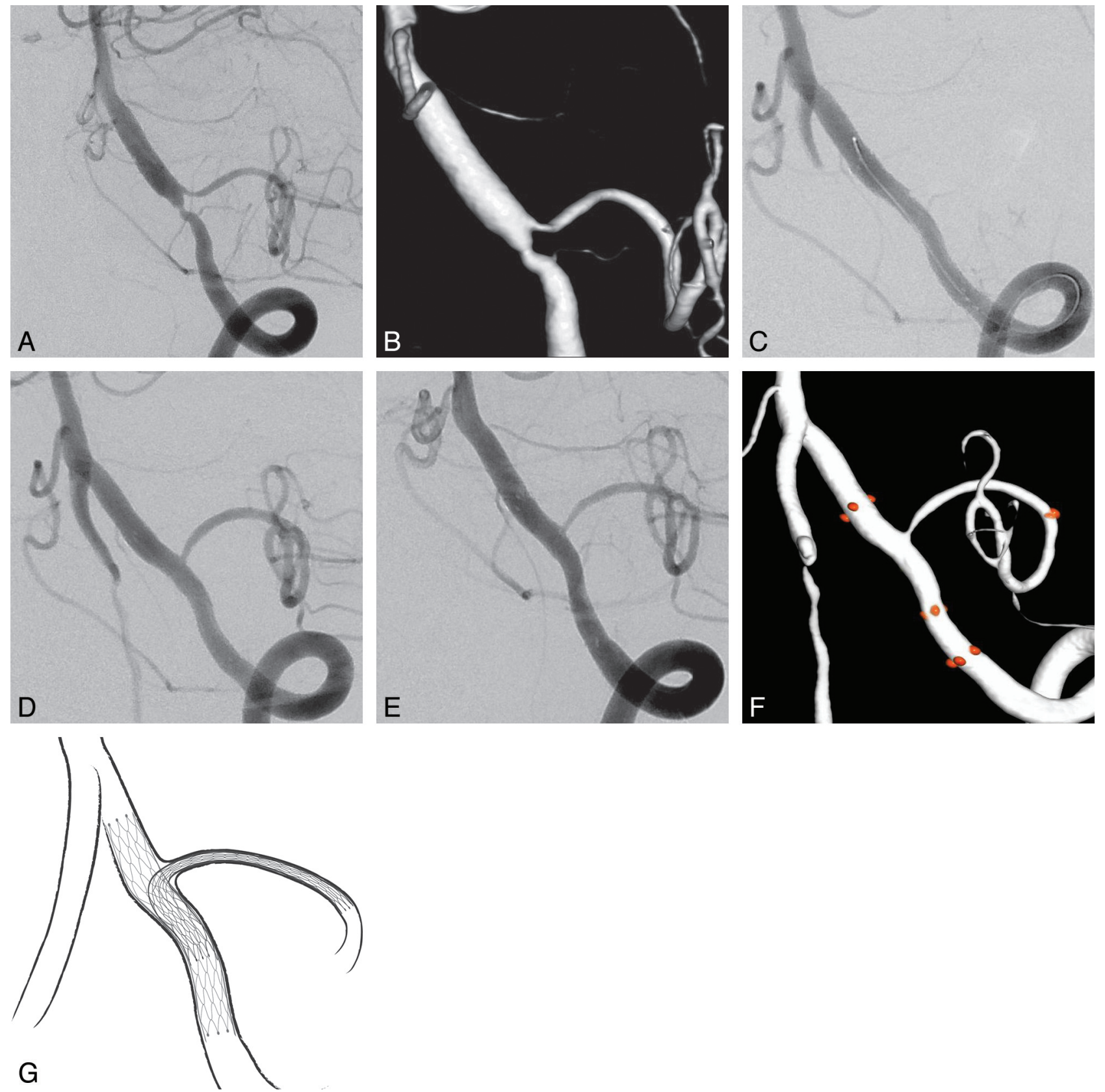

Fig 2. $A$ and $B$, The lateral image of right vertebral artery angiography $(A)$ and the $3 D$ reconstruction image $(B)$. $C$, The PICA was obstructed after the deployment of the Wingspan. $D$, The Enterprise stent was placed through the proximal vertebral artery to the PICA. E, The 6-month follow-up angiography shows good patency of the vertebral artery and the PICA. F, 3D reconstruction image shows good PICA patency. G, Illustration of the vertebral artery-to-PICA stent placement.

by the vertebral artery stent placement. Using previous experience with a vertebral artery - PICA stent, ${ }^{7}$ the authors placed an Enterprise stent in the PICA with its diameter being $<2$ $\mathrm{mm}$, even though the Enterprise stent was optimal for an intracranial artery with a diameter $>2.5-4 \mathrm{~mm}$. ${ }^{4}$ There are no reports on the feasibility and safety of the stents in small intracranial vessels of $<2 \mathrm{~mm}$ in diameter, and the longer term effects of the presence of the stent have not yet been reported. ${ }^{11}$ However, there are some reports on the safety of stents in small cerebral arteries from case series studies. Turk et $\mathrm{al}^{12}$ concluded that the use of self-expanding stents in vessel diameters $<2 \mathrm{~mm}$ could be performed safely and efficaciously for short- and intermediate-term results. In addition, Siddiqui et $\mathrm{al}^{13}$ concluded that Enterprise stents were successfully and accurately deployed in vessels of $<2 \mathrm{~mm}$ in diameter with no occurrence of stent occlusion. Zhang et $\mathrm{al}^{5}$ reported that patients had safely and successfully undergone other self-expanding stent treatments, but not with the Enterprise stent, in vessel diameters of $<2.5 \mathrm{~mm}$ with positive outcomes. All of them attempted to deploy the stent and reported cases of stent deployment in the distal anterior circulation or basilar artery-PCA with relatively larger diameters than that of the PICA.

In our cases, the EVTs with the stent were challenging in the PICA with a smaller caliber, and the midterm (6-12 months) follow-up angiography revealed that flow of the PICA was patent without stenosis or occlusion. Moreover, the caliber of the PICA increased, and the angle of vertebral 
artery-PICA junction became larger after the procedure in most of our cases, and the morphologic changes of vessels made the PICA flow better. ${ }^{7}$ The promising results of this study make stent placement in smaller cerebral arteries worthy of being addressed in more patients who cannot avoid stent placement. Long-term follow-up should be performed for estimating the patency of the PICA and verifying that the stents are not in other places and the lesion did not relapse.

\section{Conclusions}

In this small group of patients with the vertebral artery-PICA lesions involving the PICA origin, the vertebral artery-toPICA stent placement appears to be a viable treatment option for preserving PICA patency in select patients. Further follow-up and more cases are necessary to determine the full effectiveness of our encouraging results.

\section{References}

1. Lemole GM Jr, Henn J, Javedan S, et al. Cerebral revascularization performed using posterior inferior cerebellar artery-posterior inferior cerebellar artery bypass. J Neurosurg 2002;97:219-23

2. Ozkan A, Azam SA, David N, et al. The occipital artery for posterior circulation bypass: microsurgical anatomy. Neurosurg Focus 2008;24:E9

3. Ansari S, McConnell DJ, Azari H, et al. Impact of intracranial self-expanding stents in the treatment of acute ischemic stroke: efficacy and limitations. $J$ NeuroIntervent Surg Epub 2011 Feb 10
4. Peluso JP, van Rooij WJ, Sluzewski M, et al. A new self-expandable nitinol stent for the treatment of wide-neck aneurysms: initial clinical experience. AJNR Am J Neuroradiol 2008;29:1405-08

5. Zhang J, Lv X, Jiang C, et al. Endovascular treatment of cerebral aneurysms with the use of stents in small cerebral vessels. Neurol Res 2010;32:119-22

6. Lubicz B, Collignon L, Raphaeli G, et al. Solitaire stent for endovascular treatment of intracranial aneurysms: immediate and mid-term results in 15 patients with 17 aneurysms. J Neuroradiol 2010;37:83-88

7. Chung J, Kim BS, Lee D, et al. Vertebral artery occlusion with vertebral arteryto-posterior inferior cerebellar artery stenting for preservation of the PICA in treating ruptured vertebral artery dissection. Acta Neurochir 2010;152: 1489-92

8. Iihara K, Sakai N, Murao K, et al. Dissecting aneurysms of the vertebral artery: a management strategy. J Neurosurg 2002;97:259-67

9. Kakino S, Ogasawara K, Kubo Y, et al. Treatment of vertebral artery aneurysms with posterior inferior cerebellar artery-posterior inferior cerebellar artery anastomosis combined with parent artery occlusion. Surg Neurol 2004;61: 185-89

10. Ogasawara K, Kubo Y, Tomitsuka N, et al. Treatment of vertebral artery aneurysms with transposition of the posterior inferior cerebellar artery to the vertebral artery combined with parent artery occlusion: technical note. J Neurosurg 2006;105:781-84

11. Lv X, Jiang C, Li Y, et al. Clinical outcomes of ruptured and unruptured vertebral artery-posterior inferior cerebellar artery complex dissecting aneurysms after endovascular embolization. AJNR Am J Neuroradiol 2010;31: 1232-35

12. Turk AS, Niemann DB, Ahmed A, et al. Use of self-expanding stents in distal small cerebral vessels. AJNR Am J Neuroradiol 2007;28:533-36

13. Siddiqui MA, Bhattacharya JJ, Lindsay KW, et al. Horizontal stent-assisted coil embolization of wide-necked intracranial aneurysms with the Enterprise stent: a case series with early angiographic follow-up. Neuroradiology 2009;51: 411-18 